\title{
TRIMMING THE APPLE TREES IN WINTER
}

Reiner Kunze

Translated by Lori M. Fisher

With these,

I sever all the branches in me

that want to extend higher

Once more

concentrating on the buds

And on the outer branches

One must be able to climb through with a basket through the very crown of an apple tree, say

the old gardeners

And overgrown sorrow and overgrown joy

must be able to make their way through

through us 